\title{
Meniscal Root Tears-The Crippling Epidemic. Case Report and Literature Review
}

\author{
Dan Crisan ${ }^{1, *}$, Dinu Vermesan ${ }^{1,2}$, Radu Prejbeanu ${ }^{2,3}$, Bogdan Deleanu ${ }^{1,2}$ and \\ Sorin Craioveanu ${ }^{1,2}$
}

\author{
${ }^{\text {I }}$ Ist Department of Orthopedic Surgery, "Pius Brânzeu” Emergency Clinical County Hospital, ıo I. Bulbuca Blvd, \\ 300736 Timișoara, Romania; dinu@vermesan.ro(D.V.); bogdandeleanu@yahoo.com (B.D.); \\ sorynncraioveanu@gmail.com (S.C.) \\ ${ }^{2}$ Ist Discipline of Orthopedics and Traumatology, "Victor Babeș” University of Medicine and Pharmacy, \\ 2 E.Murgu Sq., 30004I Timișoara, Romania; raduprejbeanu@gmail.com (R.P.) \\ ${ }^{3}$ Premiere Hospital Timișoara, II3 Calea Aradului, 300643 Timișoara, Romania \\ *Correspondence: crisan.dan@hosptm.ro; Tel.: +40-747-025-oII
}

Submitted: 22 December 2020; Accepted: 2 February 202I; Published: 8 February 202I

\begin{abstract}
I) Background: Meniscal lesions are one of the most prevalent pathologies in orthopedics; meniscal root tears have been recognized as a specific subset with poor mid- to long-term results after standard treatment options (observation, physical therapy, arthroscopic partial meniscectomy) and frequent progression to osteoarthritis. Meniscal root repair has emerged as a new technique with promising results. We present a case report and review the current literature regarding the diagnosis, indications to repair, current techniques, alternatives and expected outcomes. (2) Case report: A 49-year-old woman, with a body mass index of 30 and no significant comorbidities or history of trauma, involved in moderate to heavy physical activity, presented with left knee pain at the internal meniscal line, which worsened over the last 4 months, despite an intra-articular steroid shot. Based on the magnetic resonance imaging (MRI), she was diagnosed with a type 2 medial meniscus posterior root tear and moderate internal compartment chondropathy (Outerbridge 2). She underwent side-to-side repair of the meniscal lesion and reattachment of the root through a bone tunnel over a button. Postoperatively, she was non-weight-bearing (NWB) for 6 weeks and with the knee immobilized at $30^{\circ}$ of flexion. At 6 months' follow-up, the patient was fully ambulant without crutches, with active knee flexion between $0^{\circ}$ and $130^{\circ}$. (3) Conclusion: Meniscal root repair is reasonably accessible. It may provide additional benefits over current treatment options when used judiciously and with an adequate surgical technique.
\end{abstract}

Keywords: meniscus; root tear; repair; arthroscopy

How to cite: Crisan, D.; Vermesan, D.; Prejbeanu, R.; Deleanu, B.; Craioveanu, S. Meniscal Root Tears-The Crippling Epidemic. Case Report and Literature Review. Timisoara Med. 2020, 2020(2), 5; doi:I0.35995/tmj20200205.

\section{Introduction}

Out of all the types of meniscal tears that have been described in the scientific literature, the posterior horn of the medial meniscus is the most prevalent. Medial meniscus root tears have been described in the scientific 
literature since 1934, but it was not until the 1990s-200os that the full spectrum of root tears along with meniscal extrusion and early and rapidly evolving degenerative changes was recognized and published [I-4].

Even though these variations of meniscal tears were identified, their clinical importance was not fully understood due to a limited amount of biomechanical data knowledge and a developing understanding in the role of the meniscus in physiological knee motion. These aspects were researched and published in the late 20oos, and the functional equivalence of the posterior root meniscectomy with a total meniscectomy was determined $[5,6]$. A connection was also made between spontaneous osteonecrosis of the knee (SONK), which was thought to be an idiopathic condition of subchondral insufficiency, and meniscal root tears $[7,8]$. There was an increased interest in fixing these types of tears, a feat made especially difficult by the anatomic characteristics of the injured portion of the meniscus.

\section{Case Report}

We present a case report and review the current literature regarding the diagnosis, indications to repair, current techniques, alternatives and expected outcomes of meniscal root tears.

\section{Patient Information}

A 49-year-old woman, with a body mass index of 30 and no significant comorbidities or history of trauma, involved in moderate to heavy physical activity (farming), presented with intense left knee pain at the internal meniscal line, which started four months before, not preceded by any trauma or forced articular move. The pain worsened progressively under symptomatic treatment, leaving her with significant limitations to her daily activity - she was able to walk only with the help of two crutches. She received an intra-articular steroid shot 6 weeks before the current presentation, which failed to control her symptoms.

The patient received Clopidogrel $75 \mathrm{mg}$ QD therapy for atrial fibrillation and had no other significant medical or surgical history. During the clinical examination, the knee was not significantly swollen, showing no laxity (Lachman I*, anterior drawer $\mathrm{I}^{*}$, negative posterior drawer and varus-valgus tests), but presented positive meniscal signs (McMurray and Apley's). Non-inflamed, non-ulcerated varicose veins were present, but there were no modifications regarding the distal pulses or superficial sensitivity.

\section{Imaging Assessment}

The patient had an outpatient knee MRI performed, which showed a positive "ghost sign" (Figures I-3) and an extruded meniscal body (Figure 4). The MRI was followed by the arthroscopic exploration of the knee, confirming the diagnosis: type 2 medial meniscus posterior root tear, Outerbridge 2 chondropathy in the medial and femoro-patellar compartments and edema of the medial tibial bony plateau, with chronic secondary synovitis. 


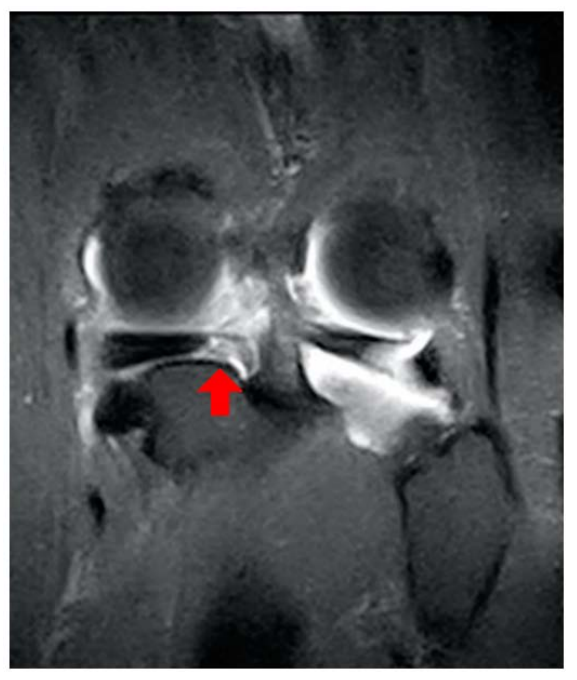

Figure 1. Coronal STIR MR image showing posterior root detachment (arrow).

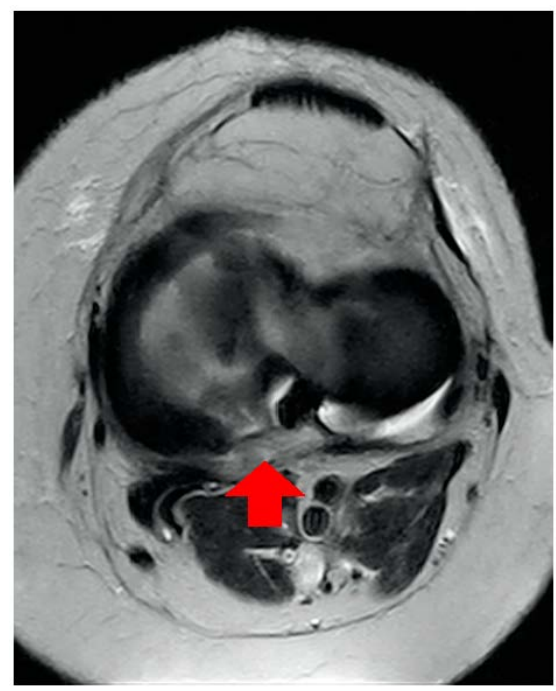

Figure 2. Sagittal T2 MR image showing the "ghost sign"-absence of the meniscus.

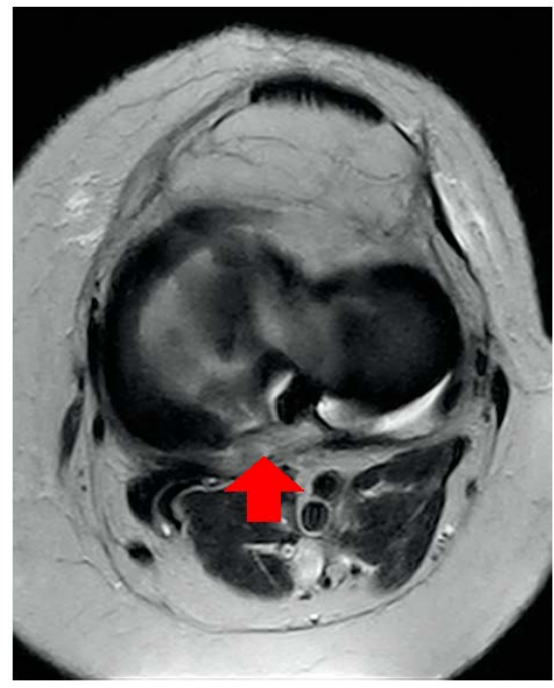

Figure 3. Axial $T_{2} M R$ image showing the posterior meniscal root detachment. 


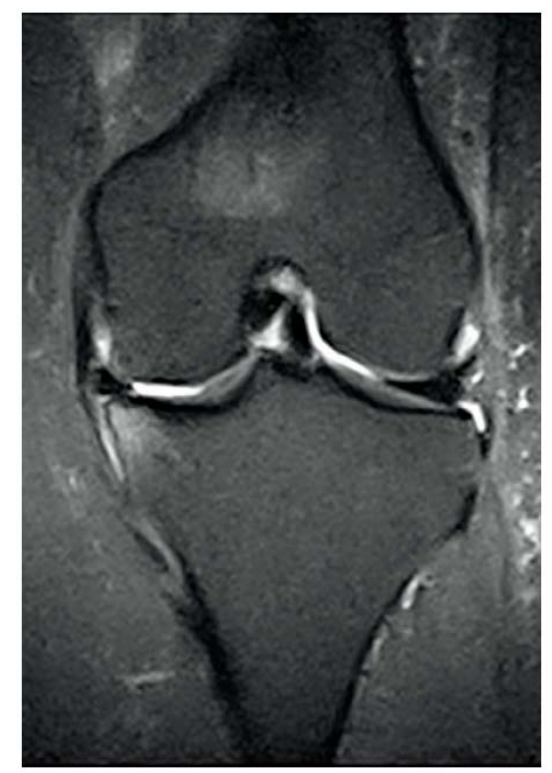

Figure 4. Coronal fast STIR MR image showing medial meniscus extrusion, medial joint space narrowing, chondromalacia and medial tibial plateau bony edema.

\section{Therapeutic Intervention}

Conservative symptomatic treatment with non-steroidal anti-inflammatory drugs was initiated, with poor response and symptom aggravation; the patient also responded poorly to an intra-articular injection with cortisone, so she was scheduled for arthroscopic medial meniscus root repair. She underwent side-to-side repair of the meniscal lesion and reattachment of the root through a bone tunnel over a button.

Intraoperatively, the type 2 tear with $>3 \mathrm{~mm}$ of meniscal insertion remaining was confirmed (Figure $5 \mathrm{~A}$ ). Superficial medial collateral lengthening (MCL) by pie-crusting was first performed to improve access to the medial compartment [9-II]. The second step of the intervention included the mobilization of the meniscus, with the determination of the physiological root insertion. A bone curette was used to remove the cartilage and a guide wire was inserted with an outside-in technique, using an Accufex Aimer Handle with the corresponding Curved Aimer Guide (Smith \& Nephew, Watford, UK), through a small ( $\sim \mathrm{Icm}$ ) incision on the anteromedial side of the proximal calf. The position of the guide wire tip was confirmed arthroscopically, and it was over-drilled with a 4 -mm cannulated drill bit up to the tibial articular cartilage. The aimer and guide wire were removed and a 2 -o monofilament non-resorbable suture was looped, inserted through the drill bit and retrieved from the joint through the medial portal with a suture grasper. The drill bit was then removed. A FirstPass Mini Suture Passer loaded with UltraBraid 2-o sutures (Smith \& Nephew, Watford, UK) was used to create the side-to side repair. The UltraBraid sutures were passed through the anteromedial portal, inserted in the monofilament loop and shuttled through the tunnel, creating the construct shown in Figure $5 \mathrm{~A}$. It was passed through a titanium button and tied securely, under arthroscopic visualization and palpation with the probe, avoiding over-constriction (Figure $5 \mathrm{~B}$ ).

\section{Follow-Up and Outcomes}

Postoperatively, the patient was NWB for 6 weeks, with the knee immobilized in a brace with $0^{\circ}-30^{\circ}$ of flexion. A course of physical therapy was then prescribed to regain full range of motion and muscular strength. At 6 months' follow-up, the patient was fully ambulant without crutches, had active knee flexion of $\mathrm{o}^{\circ}-130^{\circ}$ and presented light morning gelling and pain with overuse. 


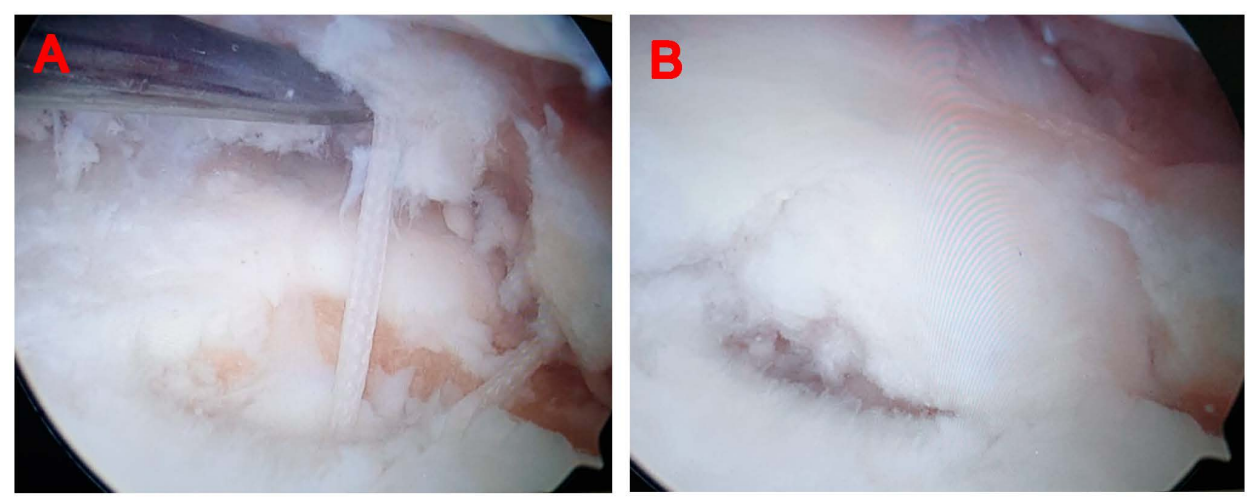

Figure 5. Arthroscopic intraoperative views: the meniscal tear, the trans-osseus tunnel and the side-to side repair (A); the finished repair $(\mathbf{B})$.

\section{Discussion}

Arthroscopic meniscectomy is one of the most frequent orthopedic surgeries performed [12,13]. However, with greater understanding of the biomechanical role of the menisci, research into meniscal vascularization, biomechanics, resection, repair, transplantation and long follow-up of large series of meniscectomized patients that showed early degenerative changes, the quintessential articular role of the menisci became obvious and a new thought model of meniscal preservation began to gain popularity with orthopedic surgeons worldwide. This was also made easier by the advent of all inside meniscal sutures that replaced classical inside-out and outside-in techniques and turned a posterior horn meniscal suture from a tedious, high-risk, high-skilled, lengthy surgery into an accessible, low-risk, medium-skilled, short procedure.

The meniscal roots were also recognized for their biomechanical importance, a root tear having a far worse outcome compared to a posterior horn tear, even if treated by conventional methods. A root partial meniscectomy was shown to have a similar effect to a total meniscectomy $[5,6]$. As described above, the diagnosis is made by corroborating the clinical examination (positive meniscal signs, little relief from conventional conservative treatment) with imaging data ("ghost sign", meniscal extrusion, SONK).

La Prade et al. classified meniscal root tears based on their morphology into five types (Table I) [14]. The advantage of this classification system is that it can be used for medial and lateral posterior root tears. Our patient had a type 2 medial meniscal root tear, which is the most common type of tear, present in about one third of meniscal root tear patients [Is].

Table 1. The La Prade classification of meniscal root tears [I4].

\begin{tabular}{ll}
\hline The "La Prade" Classification of Meniscal Root Tears \\
\hline Type I & Partial root tear \\
Type 2 & Complete root tear less than $9 \mathrm{~mm}$ from root insertion \\
Type 3 & Complete root tear associated with bucket handle tear \\
Type 4 & Oblique tear into root attachment \\
Type 5 & Root avulsion fracture \\
\hline
\end{tabular}

Treatment should be individualized for each patient. The ideal candidate for surgical repair is an active patient without significant cartilage wear and a mechanically neutral lower limb, regardless of age [16,17]. Patients 
with a high BMI $\left(>30 \mathrm{~kg} / \mathrm{m}^{2}\right)$ and those with subchondral bone defects, Outerbridge $>3$ chondropathy, varus-valgus deviation or other substantial comorbidities should be managed conservatively or by alternative techniques. These include conservative treatment (NSAIDs, physiotherapy, exercise therapy), unicompartmental knee arthroplasty (UKA) for localized degenerative disease in balanced, neutral knees, total knee arthroplasty (TKA) for advanced degenerative disease, in unstable knees and varus-valgus malalignment. Arthroscopic partial meniscectomy was comparable with conservative treatment in the review conducted by Stein et al. [18].

The aim of surgical treatment is the anatomical reduction and fixation of the root tear. A number of techniques have been described in the literature. Suture anchor fixation is a technically demanding technique that involves expensive implants. Transtibial tunnel fixation is less invasive and can use one or two tunnels for fixation. The advantage of this technique is that it is an all-arthroscopic technique, passing the suture through the meniscal root in different configurations (Mason-Allen, modified Mason-Allen, horizontal mattress), and it can be performed with a multitude of instruments adapted from the shoulder, or with meniscus-specific suture passing devices. This, in combination with the root-specific tunnel aimer, significantly shortens the operative time and delivers high-quality, reproducible surgical results [12,16-I8].

\section{Patient Perspective}

Medial meniscus root tears can cause severe pain that does not respond to oral or intra-articular anti-inflammatory or anesthetic medication. Our patient accused persistent, debilitating joint line pain, which showed only moderate short-term benefits from an intra-articular steroid-lidocaine shot and mild symptom relief from oral NSAIDs. She had to significantly alter her lifestyle and was not able to perform everyday tasks. Moreover, the patient could not undergo surgery sooner because no elective surgeries were performed at that time due to the COVID-I9 pandemic. As soon as elective surgeries were permitted, she opted for surgery with immediate postoperative pain relief. After the 6 weeks of NWB with a bracing period and one month of rehabilitation, she was back to her previous daily workloads.

\section{Conclusions}

Meniscal root repair is reasonably accessible. It may provide additional benefits over current treatment options when used judiciously and with an adequate surgical technique.

Author Contributions: Conceptualization: D.C.; methodology: D.C., D.V.; resources: S.C.; data curation: D.C.; writing-original draft preparation: D.C., D.V.; writing-review and editing: R.P., B.D.; visualization: B.D.; supervision: D.V.; project administration: D.C.

Informed Consent Statement: Informed consent was obtained from the patient, who agreed to the publication of her clinical information and images, in an anonymized fashion.

Funding: This research has received no external funding.

Conflicts of Interest: The authors declare no conflict of interest.

\section{References}

I. Burrows, H.J. Two cases of ossification in the internal semilunar cartilage. Br. J. Surg. 1934, 21, 404-410. [CrossRef]

2. Pagnani, M.J.; Cooper, D.E.; Warren, R.F. Extrusion of the medial meniscus. Arthroscopy 1991, 7, 297-300. [CrossRef] 
3. Lerer, D.B.; Umans, H.R.; Hu, M.X.; Jones, M.H. The role of meniscal root pathology and radial meniscal tear in medial meniscal extrusion. Skeletal Radiol. 2004, 33, 569-574. [CrossRef] [PubMed]

4. Costa, C.R.; Morrison, W.B.; Carrino, J.A. Medial meniscus extrusion on knee MRI: Is extent associated with severity of degeneration or type of tear? AJR Am. J. Roentgenol. 2004, 183, 17-23. [CrossRef] [PubMed]

5. Harner, C.D.; Mauro, C.S.; Lesniak, B.P.; Romanowski, J.R. Biomechanical consequences of a tear of the posterior root of the medial meniscus. Surgical technique. J. Bone Jt. Surg. Am. 2009, 91 (Suppl. 2), 257-270. [CrossRef] [PubMed]

6. Allaire, R.; Muriuki, M.; Gilbertson, L.; Harner, C.D. Biomechanical consequences of a tear of the posterior root of the medial meniscus. Similar to total meniscectomy. J. Bone Jt. Surg. Am. 2008, 90, 1922-1931. [CrossRef] [PubMed]

7. Robertson, D.D.; Armfield, D.R.; Towers, J.D.; Irrgang, J.J.; Maloney, W.J.; Harner, C.D. Meniscal root injury and spontaneous osteonecrosis of the knee: An observation. J. Bone Jt. Surg. Br. 2009, 91, 190-195. [CrossRef] [PubMed]

8. Sung, J.H.; Ha, J.K.; Lee, D.W.; Seo, W.Y.; Kim, J.G. Meniscal extrusion and spontaneous osteonecrosis with root tear of medial meniscus: Comparison with horizontal tear. Arthroscopy 2013, 29, 726-732. [CrossRef] [PubMed]

9. Gaudiani, M.A.; Knapik, D.M.; Kaufman, M.W.; Salata, M.J.; Voos, J.E.; Karns, M.R. Percutaneous Superficial Medial Collateral Ligament Release Outcomes During Medial Meniscal Arthroscopy: A Systematic Review. Artbrosc. Sports Med. Rehabil. 2020, 2, ei53-ei59. [CrossRef] [PubMed]

ıo. Han, X.; Wang, P.; Yu, J.; Wang, X.; Tan, H. Arthroscopic pie-crusting release of the posteromedial complex of the knee for surgical treatment of medial meniscus injury. BMC Musculoskelet. Disord. 2020, 21, 301-309. [CrossRef] [PubMed]

II. Jeon, S.-W.; Jung, M.; Chun, Y.-M.; Lee, S.-K.; Jung, W.S.; Choi, C.H.; Kim, S.-J.; Kim, S.-H. The percutaneous pie-crusting medial release during arthroscopic procedures of the medial meniscus does neither affect valgus laxity nor clinical outcome. Knee Surg. Sports Traumatol. Artbrosc. 2018, 26, 2912-2919. [CrossRef] [PubMed]

I2. Garrett, W.E.; Swiontkowski, M.F.; Weinstein, J.N.; Callaghan, J.; Rosier, R.N.; Berry, D.J.; Harrast, J.; Derosa, G.P. American Board of Orthopaedic Surgery Practice of the Orthopaedic Surgeon: Part-II, certification examination case mix. J. Bone Jt. Surg. Am. 2006, 88, 66o-667. [CrossRef]

I3. Molina, C.S.; Thakore, R.V.; Blumer, A.; Obremskey, W.T.; Sethi, M.K. Use of the National Surgical Quality Improvement Program in orthopaedic surgery. Clin. Orthop. Relat. Res. 2015, 473, 1574-1581. [CrossRef] [PubMed]

I4. LaPrade, C.M.; James, E.W.; Cram, T.R.; Feagin, J.A.; Engebretsen, L.; Laprade, R.F. Meniscal root tears: A classification system based on tear morphology. Am. J. Sports Med. 2015, 43, 363-369. [CrossRef] [PubMed]

I5. Bin, S.-I.; Kim, J.-M.; Shin, S.-J. Radial tears of the posterior horn of the medial meniscus. Artbroscopy 2004, 20, 373-378. [CrossRef] [PubMed]

16. Cinque, M.E.; Chahla, J.; Moatshe, G.; Faucett, S.C.; Krych, A.J.; Laprade, R.F. Meniscal root tears: A silent epidemic. Br. J. Sports Med. 2018, 52, 872-876. [CrossRef] [PubMed]

17. Pache, S.; Aman, Z.S.; Kennedy, M.; Nakama, G.Y.; Moatshe, G.; Ziegler, C.; Laprade, R.F. Meniscal Root Tears: Current Concepts Review. Arch. Bone Jt. Surg. 2018, 6, 250-259. [PubMed]

18. Stein, J.M.; Yayac, M.; Conte, E.J.; Hornstein, J. Treatment Outcomes of Meniscal Root Tears: A Systematic Review. Arthrosc. Sports Med. Rehabil. 2020, 2, e25I-e26I. [CrossRef] [PubMed]

$C_{202 \mathrm{I}}$ Copyright by the author. Licensed as an open access article using a CC BY 4.0 license.

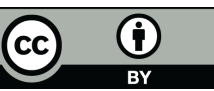

